New Mathematics and Natural Computation

Vol. 12, No. 3 (2016) 277-278

(C) World Scientific Publishing Company

DOI: 10.1142/S1793005716990015

\title{
Author Index Volume 12 (2016)
}

Agarwal, N., see Bhatt, P.

12 (2016) 241-249

Ahsanullah, T. M. G. and Al-Thukair, F., Links between probabilistic convergence groups under triangular norms and enriched lattice-valued convergence groups

Akram, M. and Waseem, N., Certain metrics in m-polar fuzzy graphs

Al-Thukair, F., see Ahsanullah, T. M. G.

Arumugam, S., Bhutani, K. and Sathikala, L., On $(r, s)$-fuzzy domination in fuzzy graphs

Bhatt, P., Agarwal, N. and Chakraborty, U. K., Parameter optimization of PEMFC with genetic algorithm

Bhutani, K., see Arumugam, S.

Boyce, M., Byrne, M., Dorpinghaus, E., Malik, D. S.

and Mordeson, J. N., Diffusion in networks: The strategic spread of Islamism

Bruun, C., Rediscovering the economics of keynes in an agent-based computational setting

Byrne, M., see Boyce, M.

Chakraborty, U. K., see Bhatt, P.

Desai, A. S., see Desai, S. S.

Desai, S. and Potdar, R., Full rationality of fuzzy choice functions on base domain through indicators

Desai, S. S. and Desai, A. S., Quasi-transitive rationality of fuzzy choice functions through indicators

Dorpinghaus, E., see Boyce, M.

Euwe, M., Mathematics - Set-theoretic considerations on the game of chess

Forouzesh, F., $N$-fold obstinate ideals in $M V$-algebras

Forouzesh, F., Some results on $M V$-modules

Malik, D. S., see Boyce, M.

Mondal, M., see Ray, K. S.

Mordeson, J. N., see Boyce, M.

Potdar, R., see Desai, S.

Ray, K. S. and Mondal, M., Logical inference by DNA strand algebra

Sathikala, L., see Arumugam, S.

Schumann, A., Towards slime mould based computer

Stefanov, S. Z. and Wang, P. P., A day-ahead wave healing in a power grid

$\mathbf{1 2}(2016) 53-76$
$\mathbf{1 2}(2016) 135-155$
$\mathbf{1 2}(2016) 53-76$
$\mathbf{1 2}(2016) 1-10$
$\mathbf{1 2}(2016) 241-249$
$\mathbf{1 2}(2016) 1-10$

12 (2016) $113-133$

12 (2016) $77-96$

12 (2016) 113-133

12 (2016) 241-249

12 (2016) 191-208

12 (2016) 175-189

12 (2016) 191-208

12 (2016) 113-133

12 (2016) 11-20

12 (2016) 265-275

12 (2016) 251-263

12 (2016) 113-133

12 (2016) 29-44

12 (2016) 113-133

12 (2016) 175-189

12 (2016) 29-44

12 (2016) $1-10$

12 (2016) 97-111

12 (2016) 45-51 
Stefanov, S. Z. and Wang, P. P., Day-ahead anticipation of complex network vulnerability

12 (2016) 209-217

Veetil, V. P., Out-of-equilibrium dynamics with heterogeneous capital goods

Vela Velupillai, K., Seven kinds of computable and constructive infelicities in economics

Velupillai, K. V., Max Euwe's set-theoretic observations on the game of chess - Introductory notes

Wang, P. P., see Stefanov, S. Z.

Wang, P. P., see Stefanov, S. Z.

Waseem, N., see Akram, M.

12 (2016) $157-173$

12 (2016) 219-239

12 (2016) 21-28

12 (2016) 45-51

12 (2016) 209-217

12 (2016) 135-155 